\title{
SOURCES INFRAROUGES À BASE de nano-antennes plasmoniques
}

\author{
Mathilde MAKHSIYAN \\ Patrick BOUCHON \\ ${ }^{1}$ III-V Lab, Nokia Bell Labs, \\ Palaiseau, France \\ Onera, The French Aerospace \\ Lab, Palaiseau, France \\ patrick.bouchon@onera.fr
}

\begin{abstract}
Un corps noir émet un rayonnement thermique sur un spectre large ne dépendant que de sa température. L'utilisation de concepts de la nanophotonique permet de modifier l'émissivité d'un objet artificiellement. En combinant des nano-antennes jouant le rôle d'émetteurs thermiques indépendants à des longueurs d'onde et pour une polarisation données, une nouvelle catégorie de sources est créée avec la possibilité d'avoir des transitions abruptes de l'émission sur une échelle spatiale correspondant à la limite de diffraction.
\end{abstract}

attrayantes dans le domaine infrarouge où les LEDs sont moins performantes que dans le visible, et les sources lasers (lasers à cascades quantiques ou oscillateurs paramétriques optiques) sont beaucoup plus onéreuses.

\section{Les propriétés des sources thermiques à nanoantennes}

\section{Rendre une surface métallique absorbante avec des nanoantennes...}

Le comportement optique d'un miroir métallique peut être modifié à l'aide de structurations de dimensions sub-longueur d'onde. Par exemple, en utilisant des bâtonnets métalliques déposés sur une bicouche isolant-métal, il est possible de rendre cette surface complètement absorbante à une longueur d'onde (voir figure 1). Ainsi, un réseau de bâtonnets d'or de $1 \mu \mathrm{m}$ de long et $100 \mathrm{~nm}$ de large, placé sur une couche de $220 \mathrm{~nm}$ de silice et un miroir d'or, va absorber toute la lumière dans le moyen infrarouge à $3,5 \mu \mathrm{m}$ pour la polarisation transverse magnétique(TM). Ce phénomène, qui peut sembler paradoxal, s'explique par une résonance dans la cavité créée entre le bâtonnet métallique et le miroir d'or inférieur, analogue à une cavité Fabry-Pérot. Cette résonance est localisée : la longueur d'onde est déterminée principalement par la longueur du bâtonnet et l'indice dans la cavité, tandis que la période ou l'angle d'incidence n'ont qu'une faible influence. possibilités d'ingénierie sont très larges,
et ces sources sont particulièrement

(a)
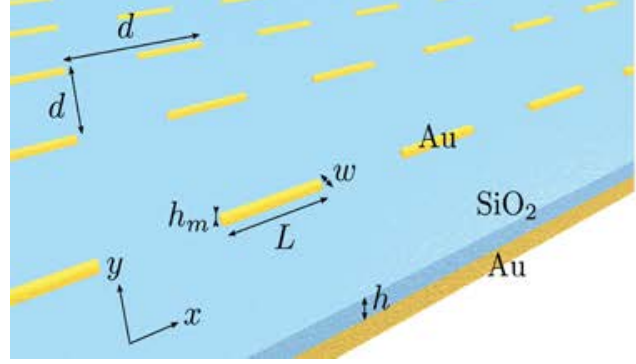
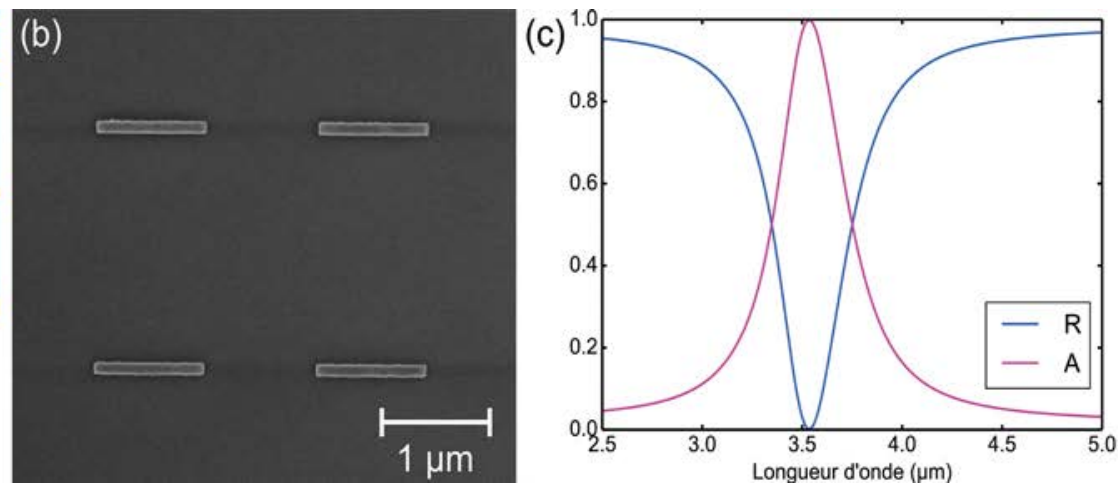

Figure 1. (a) Schéma de l'antenne MIM. (b) Image au microscope électronique à balayage (avec la permission de Christophe Dupuis (CNRS-C2N)). (c) Spectre théorique d'absorption et de réflexion d'une antenne MIM disposée périodiquement $(w=0,1 \mu \mathrm{m}, L=1 \mu \mathrm{m}$, $\left.h=220 \mathrm{~nm}, h_{m}=50 \mathrm{~nm}\right)$. 


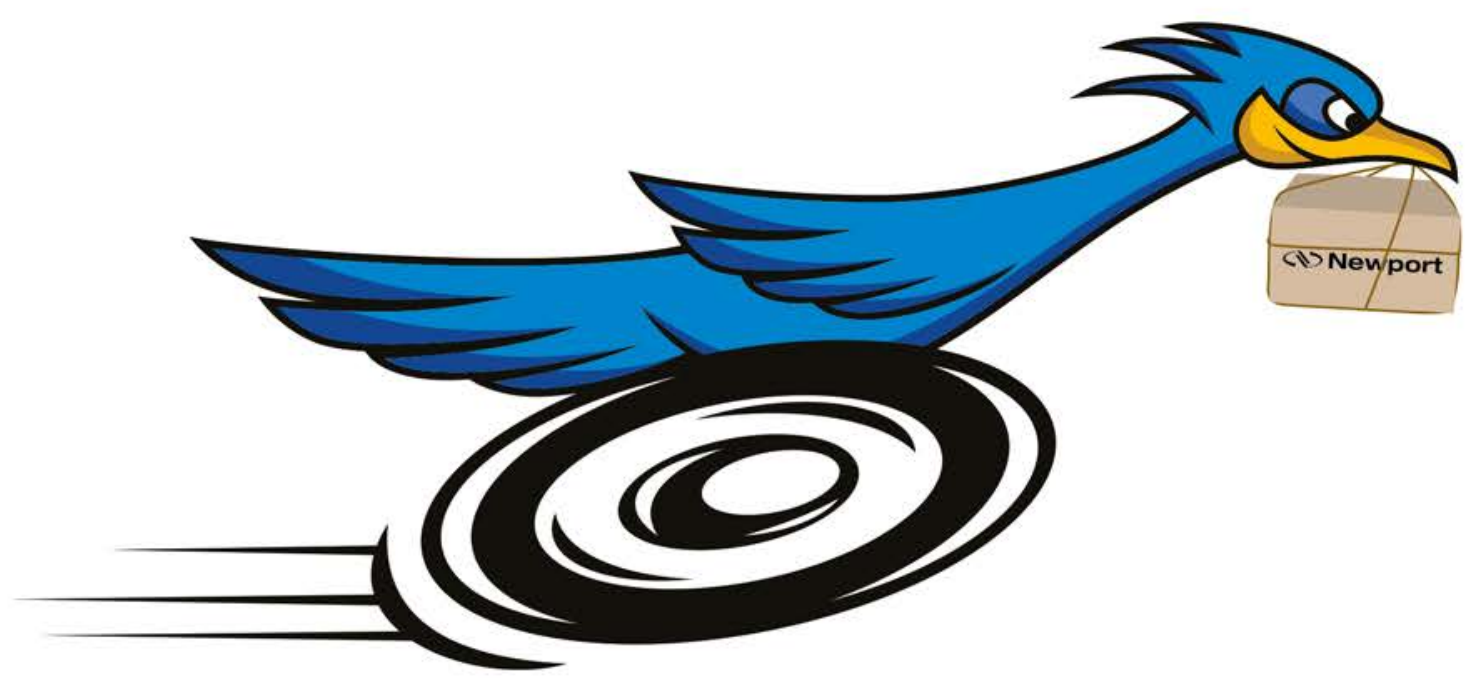

\section{La recherche prend du temps. Pas les commandes de matériel.}

\section{Des milliers de produits en stock et une livraison en 2 jours =GRATUITE*}

Equiper votre labo ou vos installations de recherche doit être rapide et efficace. Chez MKS, nous avons compris cela. C'est pourquoi nous augmentons considérablement nos stocks, nous vous proposons une nouvelle expérience d'achat en ligne simplifiée et la livraison en 2 jours GRATUITE*. L'achat de produits Newport pour votre labo vient de gagner beaucoup en facilité et en rapidité.

Un CHOIX plus rapide - Trouvez plus vite avec la recherche améliorée et des filtres

Une LIVRAISON plus rapide - Livraison en 2 jours GRATUITE* sur tous vos produits Newport préférés

Des RÉSULTATS plus rapide - L'excellente qualité des produits et de notre support technique vous ouvre la voie du succès

Soyez réactifs avec Newport. II n'y a pas de temps à perdre.

Visitez www.newport.com dès aujourd'hui

* Pour plus de détails et les conditions, consultez www.newport.com/free2day

S'applique uniquement aux commandes passées et livrées en Amérique du Nord et en Europe

\section{:mks}

@ Newport

Pour plus d'informations sur notre marque Newport, visitez www.newport.com. 
Le couplage critique (pour lequel la réflectivité est nulle ou, de façon équivalente, l'absorption est totale) est, lui, fixé par la largeur de l'antenne, l'épaisseur de l'isolant et par la densité d'antennes. Il est possible de rester proche du couplage critique pour différentes longueurs d'antennes, en gardant le même empilement de couches.

Du fait de la très faible épaisseur des couches impliquées dans ce résonateur au regard de la longueur d'onde de la lumière $(\lambda / 100$ pour l'épaisseur des antennes, $\lambda / 20$ pour l'épaisseur de la couche isolante), il est communément désigné comme une métasurface dont les briques élémentaires (les bâtonnets métalliques) peuvent être appelés méta-atomes.
Il existe différentes variantes de ce résonateur, appelé antenne MIM pour décrire l'empilement métal-isolant-métal [2]. Il a également été montré qu'on peut étendre la bande spectrale d'utilisation en combinant ces antennes [3], et qu'il est aussi possible de manipuler la phase ou la polarisation de l'onde réfléchie par cette surface.

\section{... pour faire des sources juxtaposées avec des couleurs IR choisies à façon}

L'émissivité d'un corps est liée à son coefficient d'absorption (ou absorptivité) par la loi de Kirchhoff. Ainsi, pour un corps quelconque à l'équilibre thermodynamique, son émissivité $\varepsilon$ est égale à son absorptivité a pour chaque longueur d'onde, chaque direction, chaque polarisation. En d'autres termes, un bon absorbant est un bon émetteur et réciproquement. Les métasurfaces absorbantes présentées ci-dessus deviennent alors d'excellents candidats pour la conception d'émetteurs thermiques sur des bandes spectrales étroites.

Afin d'étudier expérimentalement l'émission de ces métasurfaces, il est nécessaire de développer un banc de mesure spécifique permettant d'accéder aux propriétés spectrales et spatiales de l'émissivité. La combinaison d'un spectromètre infrarouge à transformée de Fourier (FTIR) et d'une caméra infrarouge refroidie à très haute résolution constitue un moyen de les acquérir
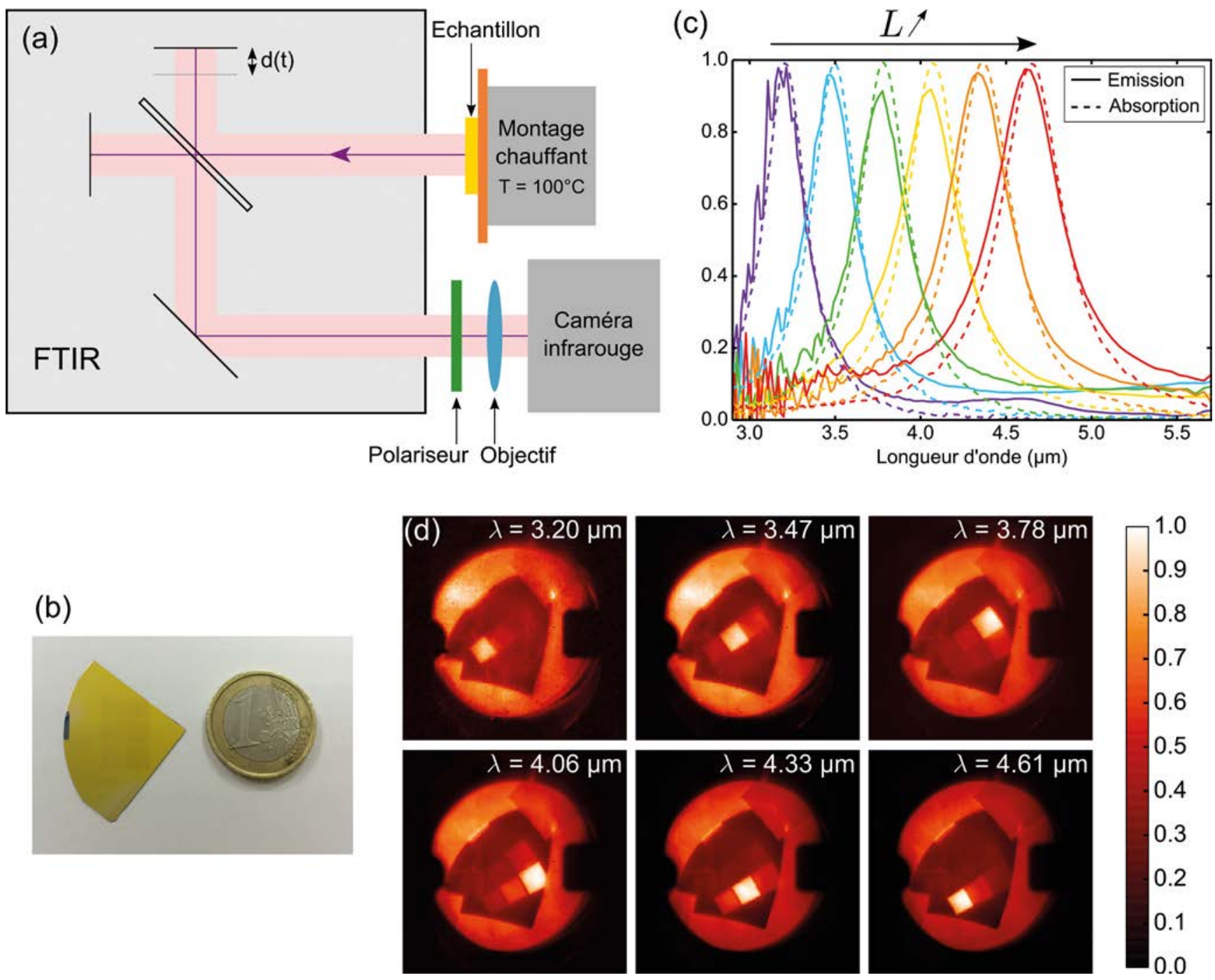

Figure 2. (a) Schéma du montage de caractérisation spatio-fréquentiel de l'émission thermique. (b) Photo de l'échantillon comportant 6 sources thermiques ayant des couleurs infrarouges différentes. (c) Spectres expérimentaux d'absorption et d'émission des 6 sources.

(d) Images de l'échantillon 6 sources à différentes longueurs d'onde. 
efficacement. La figure 2 montre le schéma d'un tel montage : l'échantillon est chauffé par un module thermo-électrique Peltier, son émission est analysée par le FTIR puis est imagée sur la caméra infrarouge. Ce banc permet ainsi d'obtenir des informations à la fois spectrales et spatiales sur l'émission des échantillons via l'acquisition couplée de spectres et d'images IR. Un polariseur peut être ajoutéa la sortie du FTIR pour connaître l'émission thermique dans chaque polarisation.

Un premier échantillon constitué de six réseaux périodiques d'antennes MIM de différentes longueurs est caractérisé avec ce montage grâce auquel on peut extraire des images de l'émission infrarouge de chacun des six réseaux à n'importe quelle longueur d'onde. Les images obtenues aux longueurs d'onde de résonance des six réseaux sont présentées à la figure 2. Chaque image a été normalisée par son maximum d'intensité. À chaque longueur d'onde de résonance $\lambda_{\mathrm{r}}$, seulement un des six réseaux émet de la lumière tandis que les cinq autres ont une faible émissivité (celle de l'or simple, proche de $1 \%$ ), certains sont même indiscernables par rapport au fond. Six émetteurs avec des réponses spectrales qui se recouvrent faiblement ont ainsi été réalisés sur un même échantillon dans le même procédé technologique.
L'étude des spectres d'émissivité expérimentaux (figure 2) confirme ce résultat. Chaque réseau possède sa propre émissivité quasi-unitaire dans la bande [3-5] $\mu \mathrm{m}$, à la longueur d'onde $\lambda_{\mathrm{r}}$ qui correspond donc à une longueur d'antenne $L$. On note également que la mesure des spectres expérimentaux d'absorption, tracés sur la même figure, est en accord avec la loi de Kirchhoff.

Les résultats obtenus avec cet échantillon montrent le potentiel de ces antennes MIM pour le contrôle spectral et spatial de l'émissivité. En nanostructurant seulement quelques pourcents d'un miroir d'or, il est possible de sculpter sa réponse spectrale. Ainsi, six émetteurs thermiques de taille millimétrique ont été réalisés sur un même échantillon, chacun possédant une émissivité quasi-unitaire, plus étroite spectralement qu'un corps noir traditionnel et à une longueur d'onde choisie entre 3 et $5 \mu \mathrm{m}$ [4].

\section{Contrôle à l'échelle de la longueur d'onde et co-localisation d'émetteurs}

Les sources thermiques à base d'antennes présentées jusqu'ici reposent sur la répétition périodique d'un motif, leurs propriétés sont donc spatialement homogènes. La modulation spatiale de l'émission à l'échelle de la longueur d'onde n'ayant jamais été réalisée, on peut se demander s'il est possible de contrôler l'émission thermique au niveau d'une cellule élémentaire de dimension proche de la longueur d'onde, voire sub-longueur d'onde.

Une métasurface inhomogène est présentée dans la suite, en jouant sur deux propriétés attrayantes de ces antennes : (i) la possibilité d'associer plusieurs antennes différentes sur une surface inférieure à la limite de diffraction, et (ii) chaque antenne supporte une résonance localisée, qui ne met pas en jeu d'effet collectif et ne dépend que très faiblement des antennes voisines.

La figure 3 montre une image $\mathrm{d}^{\prime}$ un échantillon, où une surface de $1,2 \times 0,8 \mathrm{~cm}^{2}$ contient 24 millions de cellules élémentaires de $2 \times 2 \mu \mathrm{m}^{2}$, chacune possédant sa propre combinaison d'antennes. Une image MEB de neuf cellules composées chacune d'une ou plusieurs antennes de dimensions et orientations variées est montrée en $f$ gure 3 , avec le spectre d'émissivité théorique associé. Pour chaque cellule, on a un spectre d'émissivité choisi dans les deux polarisations, indépendant des pixels voisins. Rigoureusement, l'émissivité de la métasurface doit être
Filtres Interférentiels

De 200 á 15000 nm

- Passe-bande

- Passe-haut

- Passe-bas

- Large bande

- Densité neutre

- Disponible en stock

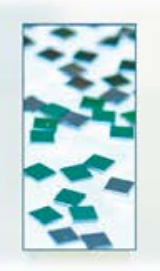

De 150 á 2000 nm

- Compression d'impulsion

- Télècom

- Accordabilité spectrale

- Monochromateurs

- Spectroscopie

- Disponible en stock

UK (parle francais): sales.uk@spectrogon.com·Tel +44 1592770000

Sweden (headquarters): sales.se@spectrogon.com·Tel +46 86382800 US: sales.us@spectrogon.com·Tel+19733311191

www.spectrogon.com 


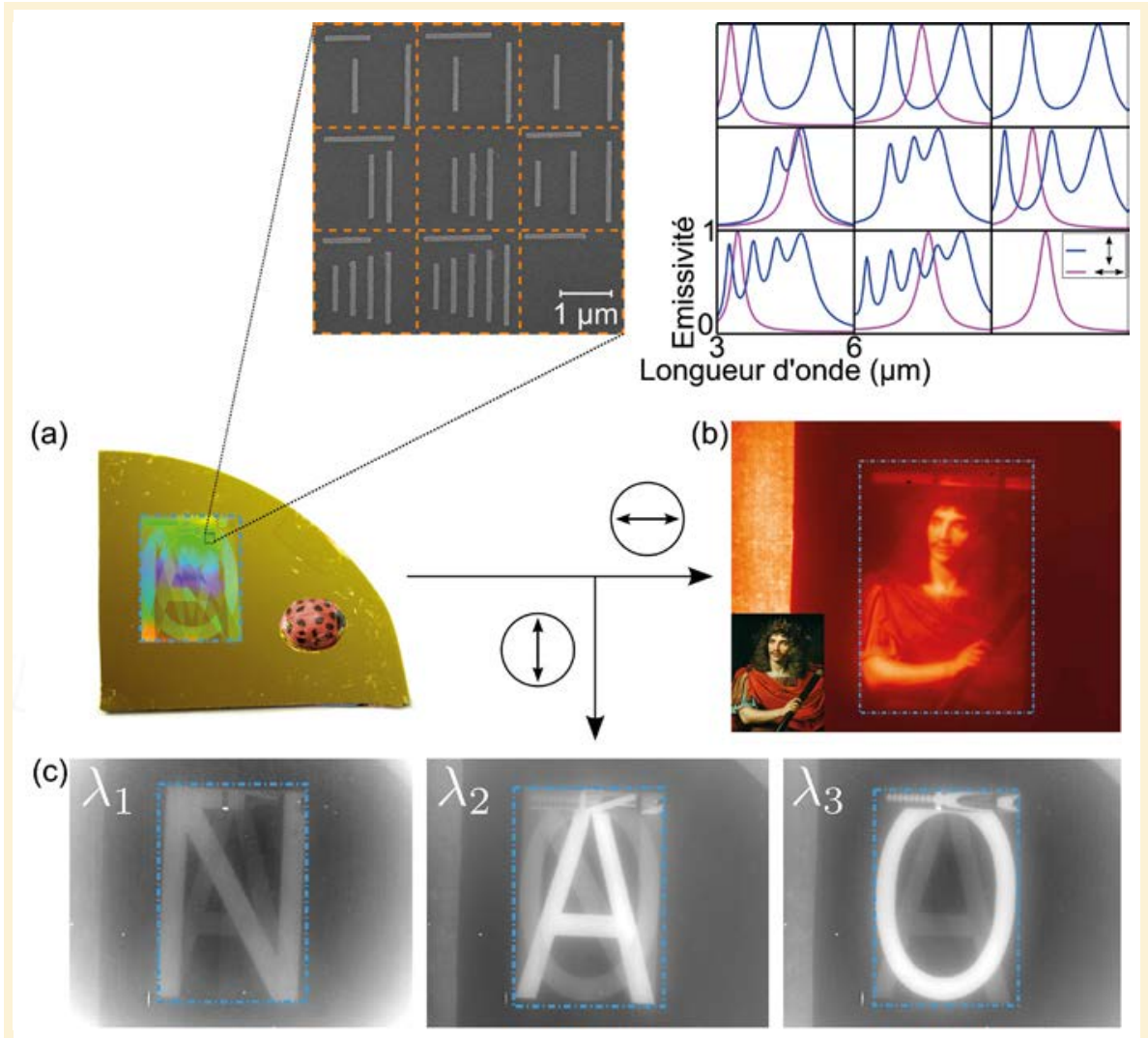

Figure 3. (a) Photo de l'échantillon, avec une image au microscope électronique à balayage d'un ensemble de 9 pixels contenant une combinaison d'antennes. Chacun de ces pixels, périodisé, a alors une émissivité où on a sculpté la réponse à façon sur la bande [3-6 $\mu \mathrm{m}$ ], indépendamment pour les 2 polarisations rectilignes. (b) Image obtenue pour la polarisation horizontale. (c) Images obtenues pour la polarisation verticale, à différentes longueurs d'onde.

considérée en utilisant la forme locale de la loi de Kirchhoff, chaque antenne codant une densité d'émissivité pour une polarisation [5].

L'échantillon est analysé avec le banc de caractérisation présenté auparavant, utilisé sans le FTIR: l'échantillon est chauffé à $100^{\circ} \mathrm{C}$ et la lumière émise est directement imagée sur la caméra infrarouge via une lentille. Différentes images d'émission infrarouge sont obtenues en fonction de la polarisation (figure 3).

Dans la polarisation correspondant aux bâtonnets horizontaux sur l'image MEB, un tableau de Molière a été codé où chaque niveau d'intensité de l'image correspond à une longueur d'antenne. Pour cela, le tableau original a été converti dans une échelle de 12 niveaux de gris et chaque niveau de gris a été codé avec une longueur d'antenne spécifique, en prenant en compte leur luminance intégrée sur la bande d'observation du détecteur [3-5 $\mu \mathrm{m}$ ] à une température de $100^{\circ} \mathrm{C}$. Ainsi, une longueur spécifique d'antenne. En ajoutant des filtres dans le montage, on peut observer les images d'émission IR de chaque lettre. Trois lettres (N, A et $\mathrm{O}$ ) sont montrées en figure 3 .

Cette métasurface inhomogène permet donc de moduler spatialement l'émissivité jusqu'à l'échelle de la longueur d'onde dans l'infrarouge et ainsi de réaliser un codage d'images multispectrales et polarisées [4].

\section{Conclusion}

Les nanoantennes plasmoniques offrent de nombreuses possibilités pour manipuler l'émission de lumière dans l'infrarouge. Il est envisageable de développer des nouvelles familles de sources émettant des « couleurs » infrarouges différentes, même si la colorimétrie n'a pas encore de référentiel reconnu comme ceux existant dans le visible. Mais cela devrait évoluer avec le développement de détecteurs infrarouges multispectraux. Ces sources sont très prometteuses pour les applications qui requièrent un spectre d'émission étroit.

Enfin, la possibilité de générer de manière contrôlée des transitions abruptes d'émissivité peut servir d'outil pour tester la capacité d'un système optique à restituer les détails d'une image, mais aussi pour encoder des images cachées à des fins de marquage de suivi ou pour la lutte anti-contrefaçon. Ces résultats préfigurent aussi le développement d'afficheurs infrarouges où l'émissivité de chaque pixel est pilotable, et qui serait l'alter ego des écrans visibles à base de LEDs. 